\title{
Polypyrrole Micro/Nanostructure Prepared Using Azo Dyes with Different Substituents
}

\author{
Dusan Kopecky, Jitka Skodova, Martin Vrnata, Premysl Fitl \\ Department of Physics and Measurements, Institute of Chemical Technology, Prague, Czech Republic \\ Email: kopeckyd@vscht.cz
}

Received 2012

\begin{abstract}
This contribution deals with simple way of polypyrrole structure modification. Using azo dyes in polymerization reaction as soft-template with similar molecular structure but different type and distribution of substitution groups lead to formation of one-dimensional and newly also three-dimensional polypyrrole micro/nanostructures. These structures are characteristic with geometrical symmetry and uniformity. Geometry of prepared structures was studied by scanning electron microscopy (SEM) and by methods of image analysis; nanotubes are hundreds of $\mathrm{nm}$ in diameter and units of $\mu \mathrm{m}$ in length, new tree-dimensional structures have units of $\mu \mathrm{m}$ in diameter. Infrared spectra (ATR-FTIR) confirmed that azo dyes work only as intermediate supporting structures without reaction with polypyrrole.
\end{abstract}

Keywords: Polypyrrole; Nanostructure; Azo Dye; Soft-template

\section{Introduction}

Conducting polymers (CP) are popular organic semiconducting materials which have been attracting attention since discovery of their electrical conductivity in the 1970s. Usually the conductivity of CP is around tenths or units of $S \cdot \mathrm{cm}^{-1}$ [1] and charge transfer is predominantly directed by variable range hopping mechanism due to highly disordered physical structure with predispositions to slowly self-degradation of conjugation by atmospheric oxygen or water vapors. For some application as plastic electronic, sensors or actuators there should be better to dominate intrinsic band conductivity with long term stability [2].

Last decade of intensive research showed some remarkable properties of CP - namely their ability to organize themself under certain conditions into highly ordered systems. CP are able to create uniform structured shapes with micrometric or even nanometric sizes (nanostructures), for example nanotubes, nanowires, nanorods [3]. This could be the potential future solution of their designated disadvantages and further, there is possibility to obtain material with higher specific surface.

These known shapes are often collectively called 1-D structures of conducting polymers. The description is based on a widely used simplification which assumes that structural properties of 1-D polymer structure are predominantly determined by its longest dimension (which is always orders of magnitude larger than the other two).

1-D structured CP could be synthesized using either template or special template free procedures. There are two means of template synthesis, the so-called hard and soft template method. The hard template method uses zeolites and membranes as a hard template, but the necessity of template removing after synthesis is very limiting.

On the other side the so called soft-template synthesis, using intermediate supporting structures of auxiliary substances (mi- cellar systems, surfactants etc.), brings many advantages: (i) change in temperature synthesis, time synthesis or molar ration of reactants can influence the geometric dimension of the prepared 1-D structures, (ii) it is effective, cheap and simple method, (iii) template autonomously degrades after the reaction is over and therefore it is easily removed from solution without damaging of prepared polymer nanostructure [4].

Soft-template method presented here is based on supposed reaction of azo dye and oxidant which create temporary supporting structure. After addition of the monomer (in our case pyrrole), polymer (polypyrrole) forms on its surface resulting in less or more ordered systems. Some of them should be considered as 3-D structures thanks to their spatial symmetry. All uses only two basic types of azo dye molecular structures as a soft-template; substantial difference is in the type of azo dye substituents and their position on the skeleton of molecule.

The molecular structure of azo dyes significantly affects the structure of prepared structured polymer. Therefore it must be reflect strength, number and distance of ligands in a molecule of azo dye, acidobasic properties, degree of molecule planarity, etc.

\section{Experimental}

Pyrrole, ferric chloride $\left(\mathrm{FeCl}_{3}\right)$, Methyl Orange, Methyl Red, Congo Red, Acid RED 1, Orange G, Sunset Yellow FCF and Tropaeolin O Sodium Salt (all purchased from Sigma-Aldrich) were used as received without further modifications. The molecular structures of azo dyes are shown in Figure 1.

The synthesis process of the structured PPY was as follows: ferric chloride (oxidant) was dissolved in $200 \mathrm{ml}$ of $5 \mathrm{mM}$ solution of azo dye and deionized water. Then $700 \mu \mathrm{l}$ pyrrole monomer was added dropwise in the first two hours of synthesis to the solution. Molar ratio of reactive monomer : oxidant : azo dye was 10:10:1 for all synthesis. The solution was tempered at 
$5^{\circ} \mathrm{C}$ and stirred during synthesis at constant speed. Due to complex structure of prepared PPY, remnants of a template must be removed by Soxhlet extraction. The prepared PPY was extracted with ethanol until extraction reagent was colorless (up to one week). The prepared structures of PPY were dried at $45^{\circ} \mathrm{C}$ in vacuum drier.

For comparison unstructured PPY was also synthesized. Molar ration of reactants (oxidant: monomer) was 1:1 in aqueous environment.

The structures of prepared PPY were observed by Scanning Electron Microscope (SEM) JEOL model JSM-7500F. Structure of prepared PPY was confirmed by Attenuated Total Reflection Fourier Transform Infrared spectroscopy (ATR-FTIR) BRUKER IFS 66v with diamond ATR attachment.

\section{Results and Discussion}

Characteristic shape of unstructured PPY is shown on SEM image Figure 2(a). This sample was synthesized by standard chemical polymerization without presence of azo dye. As seen, unstructured PPY has characteristic fruticose formations that create highly disordered arrangement.

Figure 2 (b)-(h) show SEM images of structured PPY prepared by the soft-template method using different azo dyes. It is apparent that azo dyes, as an auxiliary substance, significantly affect the structure of prepared PPY. The most evident is this capability in the case of Methyl Orange, Acid Red 1 and Sunset Yellow. In all these cases there are created symmetrical micro/nanostructures. Methyl Orange and Acid Red 1 contribute to the formation of 1-D PPY structure. Dimension of these PPY nanotubes are approximately 80 nanometers in diameter and

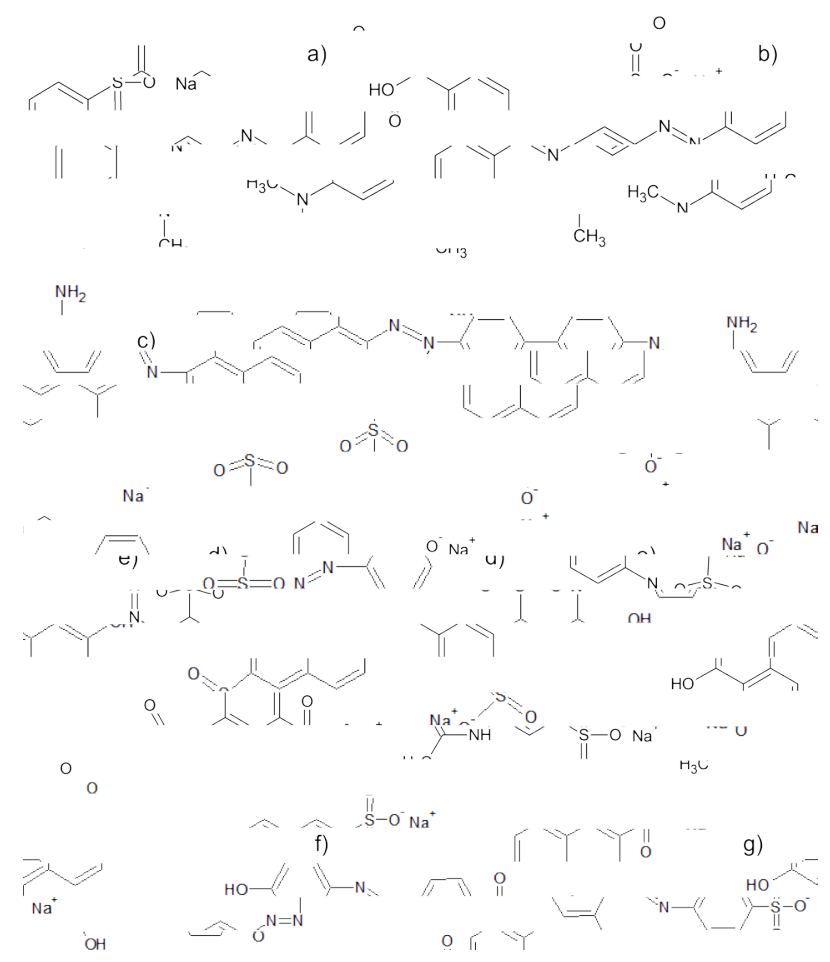

Figure 1. Chemical structures of used azo dyes: a) Methyl Orange, b) Methyl Red, c) Congo Red, d) Acid RED 1, e) Orange G, f) Sunset Yellow FCF, g) Tropaeolin O Sodium Salt.

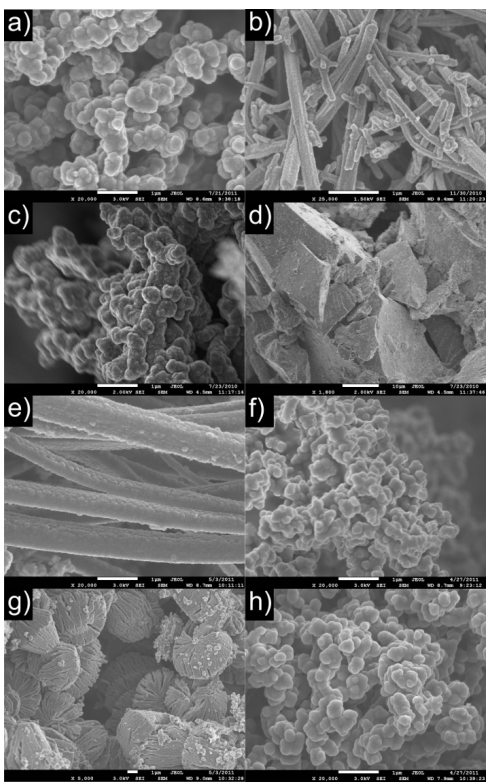

Figure 2. Structures of PPY prepared by: a) without azo dye, b) Methyl Orange, c) Methyl Red, d) Congo Red, e) Acid RED 1, f) Orange G, g) Sunset Yellow FCF, h) Tropaeolin O Sodium Salt.

hundreds of nanometers in length. Dimension of PPY nanotubes prepared from Acid RED 1 are approximately 340 nanometers in diameter and units of microns in length.

PPY synthesized in presence of Sunset Yellow FCF creates brand new type of formations. There are observed symmetrical 3-D structures. Probably, the Sunset Yellow FCF molecule allows creating more spatially-oriented complex of supporting structure in polar solvent, due to suppressing of hydrophilic and hydrophobic molecule polarization by $\mathrm{SO}_{3}{ }^{-}$polar groups.

Other azo dyes with the same molecular structure - Orange G, Tropaeolin O - are more polarized due to spreading out of polar substitution groups on and in polar solvent they are probably forced to create poorly soluble phase which suppress supporting structure formation. In this case PPY has the same structure as unstructured sample. Last sample prepared in Congo Red is comprised from "crushed stone" like formations.

Further important observation concerns degree of structure spreading in the whole volume of native liquor. Methyl Orange is the only (from all tested azo-dyes) soft-template which creates micro/nanostructures in the entire volume and also at different molar ratios $(10: 10: 1,10: 10: 0,5,10: 10: 0,1)$. On the other side Acid RED 1 and Sunset Yellow FCF create nanostructures in insulated spatially distributed domains at higher molar ratio only (10:10:1). This is probably connected with the molecular structure of azo dye which affect still poorly understand mechanism of supporting structure creation.

The main task of ATR-FTIR measurements was to verify the chemical structure of prepared PPY and to detect potential residue of azo dye used during synthesis. Interpretation of the spectra was focused on the region $1800-600 \mathrm{~cm}^{-1}$, the so called "finger print area". Interpretation of PPY spectra could be difficult because of variable degree of conjugation of PPY backbone and a large number of different types of disturbances (non-linear shape of the polymer chain, oxidation by air oxygen, etc.). Nevertheless, in all cases it was confirmed PPY as a basic 


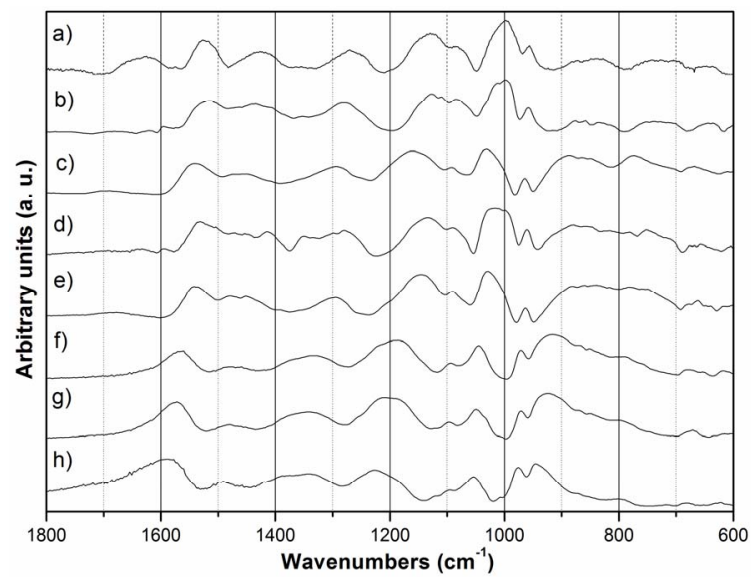

Figure 3. Overview of ATR-FTIR spectra of PPY prepared in the presence of: a) Methyl Orange, b) Methyl Red, c) Congo Red, d) Acid Red 1, e) Orange G, f) Sunset Yellow FCF, g) Tropaeolin O Sodium Salt.

unit of all formations (Figure 3). The unstructured PPY spectrum contains following characteristic peaks: $1527 \mathrm{~cm}^{-1}$ (C-C, $\mathrm{C}=\mathrm{C}$ stretching), $1427 \mathrm{~cm}^{-1}(\mathrm{C}=\mathrm{C}, \mathrm{C}-\mathrm{N}$ stretching $), 1270 \mathrm{~cm}^{-1}$ (C-H, C-N in plane deformation), $1129 \mathrm{~cm}^{-1}$ (breathing vibration of the PY ring), $1086 \mathrm{~cm}^{-1}(\mathrm{C}-\mathrm{H}, \mathrm{N}-\mathrm{H}$ in plane deformation), $997 \mathrm{~cm}^{-1}$ (C-H in plane deformation), $956 \mathrm{~cm}^{-1}$ (C-C out of plane deformation), $849 \mathrm{~cm}^{-1}$ and $727 \mathrm{~cm}^{-1}$ (C-H, N-H out of plane deformation), $646 \mathrm{~cm}^{-1}$ (C-C out of plane deformation). These peaks are evident in presented spectra of structured PPY. Slight shifts in spectra cannot be attributed to direct chemical reaction between pyrrole and azo dye, but only to changes in degree of conjugation of polypyrrole chains. It is apparent that used azo dye acts only as a supporting structure for forming polymer structure and after polymerization it self-degrades.

\section{Conclusion}

This contribution deals with preparation of PPY micro/nanostructures by soft-template method. As a soft-template seven different azo dyes were used. Using Methyl Orange and Acid RED 1 lead to formation of 1-D structures. New type (3-D structure) of PPY structure was observed for Sunset Yellow as soft-template.

\section{Acknowledgements}

This work was supported by Grant Agency of the Czech Republic (GAČR) projects No. P108/11/1298 and P108/12/P802 and also financial support from specific university research (MSMT No. 21/2012).

\section{REFERENCES}

[1] N. V. Blinova, J. Stejskal, M. Trhová, J. Prokeš, M. Omastová, "Polyaniline and polypyrrole: A comparative study of the preparation", Eur. Polym. J., vol. 43, pp. 2331-2341, 2007.

[2] U. Lange, N. V. Roznyatovskaya, V. M. Mirsky, "Conducting polymers in chemical sensors and arrays", Anal. Chimica Acta, vol. 614, pp. 1-26, 2008.

[3] X. Yang, Z. Zhu, T. Dai, Y. Lu, "Nanotubes via a reactive self-degraded template", Macromol. Rapid Commun.vol. 26, pp. 1736-1740, 2005.

[4] D. Zhang, Y. Wang, "Synthesis and applications of one-dimensional nano-structured polyaniline: An overview", Mater. Sci. Eng., B, vol. 134, pp. 9-19, 2006. 